\title{
Derma Net: An Automated Skin Lesion Analyzer Using CNN with Adaptive Learning
}

\author{
Santhi H, Gopichand G, K.Pavan Koushik, A.Nithin Krishna, D. Sai Tharun
}

\begin{abstract}
In this paper we are going to develop an automated skin lesion analyzer that can take affected skin lesion image from user and predict or approximate 3 skin diseases with 95\% accuracy. To accomplish this goal we are going to use Neural Networks as they are the best data driven models with top most accuracy in all the fields they have been experimented till now. Since Neural Network models also need huge computation power to train the model on the input data and also to predict the output we are going to use a computationally less intensive architecture that can work even on hand held mobiles and embedded systems. To further featuring our model we have added dropout techniques for model regularization and adaptive learning rates to achieve global minima with ease even with the presence of plateaus. At last we will deploy a production level web application to serve users across the world.
\end{abstract}

Keywords: Embedded Systems, Neural Networks, Global

Minima, Plateaus, Adaptive Learning Rates.

\section{INTRODUCTION}

Our proposed system for this project is to use a deep learning architecture which is more suitable for mobile and embedded based vision applications where there is lack of computation power and then convert the whole model into tensorflowjs format to deploy in the production environment so that users can access the end product any where around the world. Our application also considers user's privacy concerns as our model runs locally and any data that user submits never leaves his mobile or personal computer.

Diagnosis of skin diseases is a problem on which research is going on since last 5-10 years. People have tried so many approaches to solve this problem. Solving this problem using image processing techniques by feature extraction and segmentation is proposed in [5], since this is method is related to fine tuning it is not so reliable process to detect skin diseases as the relation between skin and its diseases can not be catched with fine tuned models. Using 3 layer neural network model which takes inputs as colour, area, shape and other hard typed properties is proposed in [6] which also need human assistance to get reliable inputs so this lacks for complete automated system for diagnosis.in recent years some came up with the idea of using pretrained image networks which are previously used for general image classification to classify this skin diseases this carried out by freezing all the hidden layers and adding one or more

Revised Manuscript Received on April 12, 2019. Institute of Technology, Vellore, T.N, India.

Gopichand G, School of Computer Science and Engineering,Vellore Institute of Technology,Vellore, T.NIndia.

K.Pavan Koushik, School of Computer Science and Engineering,Vellore Institute of Technology, Vellore, T.NIndia.

A.Nithin Krishna, School of Computer Science and Engineering,Vellore Institute of Technology,Vellore, T.NIndia.

D. Sai Tharun, School of Computer Science and Engineering,Vellore Institute of Technology,Vellore, T.NIndia.
Santhi H, School of Computer Science and Engineering,Vellore

fully connected layers at the end of this gigantic networks[7][3] even though this produced appreciable results this model does not capture the nature of disease as the classification of general objects and skin diseases are two complete different scenarios. Another paper stated to use variational auto encoders as the classifying model[4] but they are confined to detect the anomaly score which only tells the severity of infection and does not predict the type of infection that the user is facing.

\section{DESIGN APPROACH AND DETAILS}

Our proposed system comprises of data preprocessing stage, training stage, evaluation stage. During data preprocessing stage we remove duplicates from obtained image data set and augment the existing image set for better accuracy rates. In model training stage we have built input pipelines and customize mobile net by adding dense layers for classification of skin disease and adding adaptive learning techniques for faster approach to global minimum. In evaluation stage we generate report with confusion matrix, training curves with different top accuracies.

\section{A. OVERVIEW OF SYSTEM EXECUTION}

Our application aims to accept skin lesion images from users who are suffering from various skin diseases and can diagonise the image in a fraction of seconds with output showing probabilities of skin diseases [ Melanocytic nevi, Melanoma, Benign keratosis, Basal cell carcinoma, Actinic Keratoses, Vascular skin lesions, Dermatofibroma] that may been the cause for the skin lesions on patient's skin.

We have approached with a deep learning algorithm that includes convolutional neural networks and residual networks and to minimize the loss function of the algorithm with respect to time we have used adaptive learning strategy so that we can obtain global minima of the loss function in comparatively less time. And finally we have used our trained model in our website to deploy and serve it online.

\section{B. DETAILED DESIGN}

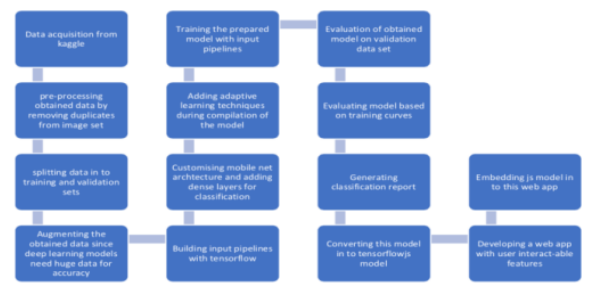

Fig.1.Explains work flow of the project during development stage

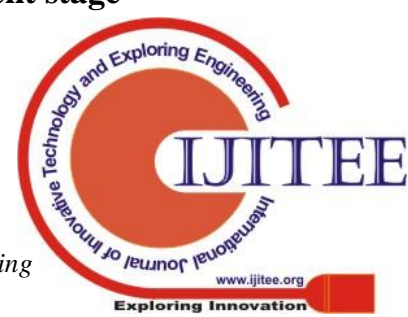




\section{ALGORITHM}

STEP1: The first step to this project is to collect reliable data for training our deep learning model and we have preferred data about skin lesions that is provided in kaggle data science web site.

STEP2: Pre processing the acquired data for preventing future run time exceptions while training the model

STEP3: Divide the data into training and testing part. Now augmentation of data has to done to increase the size of the training data as the obtained data from kaggle is not enough for training the deep network model.

STEP4: Prepare a convolutional model to predict the required outputs. As this application is meant for commodity level devices we preferred MobileNet model to train on our dataset.

STEP5: Train the model and valuate it on the validation set and finally evaluate the model based on the learning curves obtained during training.

STEP6: Convert this model in tensorflow js model to deploy it into the web application.

STEP7: Now finally develop a web application that can take input as skin image and display disease probabilities using js model we include in it.

\section{WORKING MODEL LINK}

http://dermanet.herokuapp.com/index.html

\section{E. CONSTRAINTS}

Our application is only limited to 6 skin diseases and we have worked on only 5000 images for each disease so this may have bad prediction accuracy after few years. We have only used single architecture for this model as we are limited from computational power.

\section{EXPERIMENTAL RESULTS}

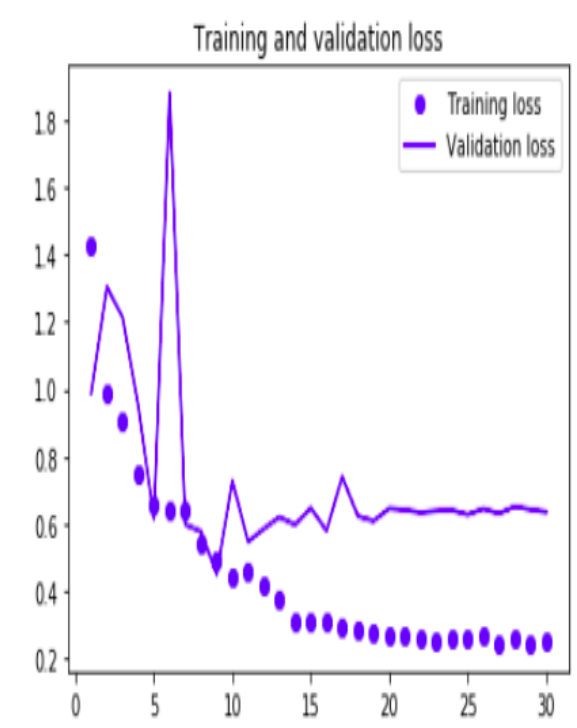

Fig.2 X-axis no.of iterations $\mathrm{Y}$-axis Training and validation loss

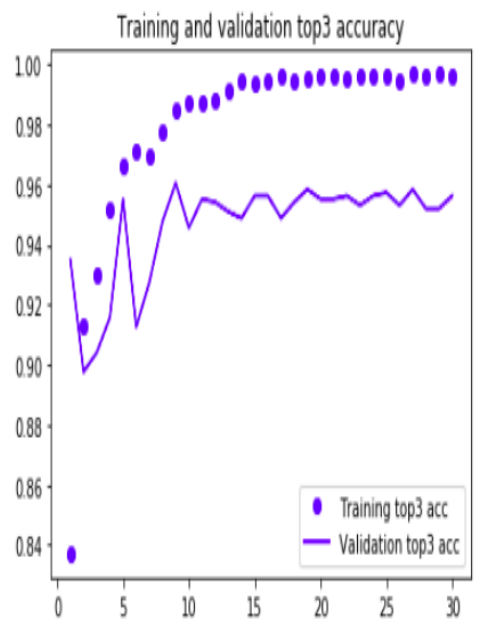

Fig.3 X-axis no.of iterations $\mathrm{Y}$-axis Training and validation loss

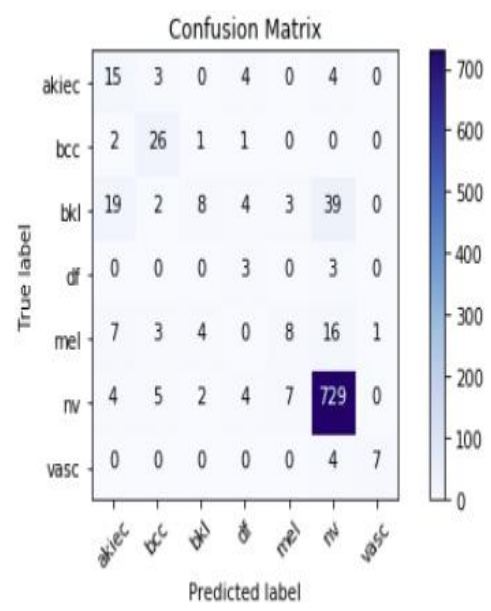

Fig.4 It is a performance table $\mathrm{X}$-axis True label $\mathrm{Y}$-axis predicted label

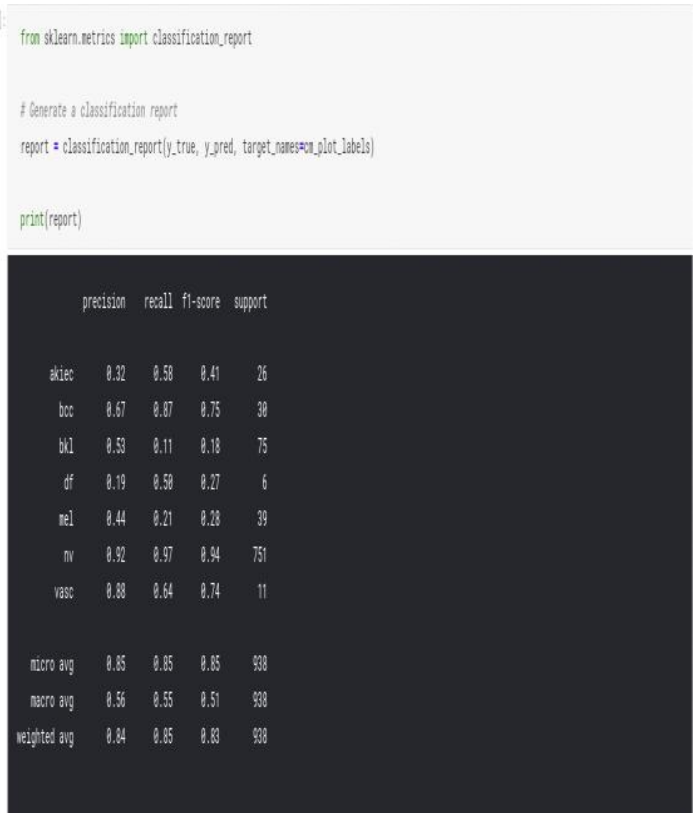

Fig.5. Classification report of the project 


\section{CONCLUSION}

With this application we are going to provide a end to end web application which can acquire skin lesion images from affected people and classify skin disease with a 95\% accuracy. But our application is only limited to 6 skin diseases and we have worked on only 5000 images for each disease so this may have bad prediction accuracy after few years. We have only used single architecture for this model as we are limited from limitation power. In future this can be enhanced with ensembling of various machine learning models with much more skin disease classes and vast data.

\section{ACKNOWLEDGMENT}

A project provides us an opportunity to have a greater understanding of the subject and explore beyond the book. On this occasion, we would like to express our heartfelt gratitudeto our faculty, Prof.G.Gopichand for his guidance andencouragement throughout this project.

Additionally, we are grateful to the VIT University Management and our School Dean for giving us an opportunity to carry out this project. It has enhanced our practical knowledge and exemplified the process of making an engineer.

\section{REFERENCES}

1. Y. LeCun, B. Boser, J. S. Denker, D. Henderson, R. E. Howard, W. Hubbard, L. D. Jackel, Backpropagation Applied to Handwritten Zip Code Recognition; AT\&T Bell Laboratories

2. LeCun, Yann."LeNet-5, convolutional neural networks". Retrieved 16 November 2013

3. Haofu Liao, Yuncheng Li, Jiebo Luo "Skin Disease Classification versus Skin Lesion Characterization:Achieving Robust Diagnosis using Multi-label Deep Neural Networks" 9 Dec 2018

4. Yuchen $\mathrm{Lu}$ and Peng $\mathrm{Xu}$ "Anomaly Detection for Skin Disease Images Using Variational Autoencoder" 24 July 2018

5. Sumithra R, Mahamad suhil, D.S. Guru "Segmentation and Classification of Skin Lesions for Disease Diagnosis" doi: 10.1016/j.procs.2015.03.090

6. Rahat Yasir,Md. Ashiqur Rahman,and Nova Ahmed,"Dermatological Disease Detection using Image Processing and Artificial Neural Network"8th International Conference on Electrical and Computer Engineering

7. Sinno Jialin Pan and Qiang Yang Fellow, "A Survey on transfer learning” IEEE 2009,10.1109/TKDE.2009.191. 